\title{
Europäische Betriebsräte: Was lehrt der deutsche Fall für die Revision der EU-Richtlinie?
}

\author{
Stefan Lücking \\ Rainer Trinczek \\ Michael Whittall
}

Bei der Umsetzung der EU-Richtlinie über Europäische Betriebsräte (EBR) sind in der Praxis Schwierigkeiten aufgetreten: Viele europaweit operierende Unternehmen und Unternehmensgruppen haben keinen EBR. Auch deshalb wird eine Revision derzeit in Konsultationen der EU-Kommission mit den europäischen Sozialpartnern verhandelt. Allerdings liegen über die Umsetzungsformen in der Praxis bisher noch nicht einmal auf nationaler Ebene ausreichende Informationen vor. In welchem Umfang konnten EBR in deutschen Unternehmen eingerichtet werden? Wo zeigten sich dabei besondere Problemzonen, die bei der Reform berücksichtigt werden sollten? 1

\section{Einleitung}

Die EU-Richtlinie über Europäische Betriebsräte vom 22. September 1994 (EBRRichtlinie) stellt einen Durchbruch im Bereich der Europäischen Sozialpolitik dar, der als wegweisend für die Entwicklung der Arbeitsbeziehungen in Europa angesehen werden kann. Nach zwei Jahrzehnten zäher Verhandlungen mit mehreren gescheiterten Anläufen war dieser Durchbruch nur gelungen, weil die Richtlinie ein neuartiges Konzept verfolgt: Sie löst das Problem unterschiedlicher institutioneller Traditionen in den einzelnen Mitgliedsländern, indem sie zwar einen gesetzlichen Rahmen für einen EBR schafft, die genaue Ausgestaltung aber einem besonderen Verhandlungsgremium in den betroffenen Unternehmen überlässt. Schon aufgrund dieser innovativen Form der Richtlinie erklärt sich, dass Artikel 15 der Richtlinie die Europäische Kommission beauftragt, spätestens zum 22 . September 1999 die Umsetzung der Richtlinie zu überprüfen und „erforderlichenfalls entsprechende Änderungen vorzuschlagen“.

Seit 1999 werden deshalb Verhandlungen und Diskussionen über eine Revision der Richtlinie geführt - bislang ohne konkretes Ergebnis. Erst Mitte Februar 2008 hat die Europäische Kommission die entscheidende zweite Phase der Konsultation über die Revision der Richtlinie eingeleitet und konkrete inhaltliche Vorschläge vorgelegt. Eines von drei Themen, die im Rahmen der Revision behandelt werden sollen, sind Maßnahmen, die Probleme und rechtliche Unklarheiten bei der Einrichtung von EBRs lösen.
Deswegen scheint es sinnvoll, zunächst zu klären, wie viele der unter die Richtlinie fallenden deutschen Unternehmen tatsächlich einen EBR haben (Abschnitt 2). Dabei werden wir auch darauf eingehen, auf welche erheblichen methodischen Schwierigkeiten man bei der Erhebung dieser Daten stößt. Diese Schwierigkeiten verweisen nun nicht nur auf spezifische Forschungsprobleme, sondern sind gleichzeitig Hinweise auf reale Probleme der Praxis. Sie werden an drei Beispielen demonstriert: der Frage von Unternehmensübernahmen und -fusionen, den uneinheitlichen EBR-Strukturen in manchen Unternehmen sowie dem spezifischen Problemfeld „Eigentümerunternehmen“ (Abschnitt 3). Der Beitrag endet mit Schlussfolgerungen für die Revision der Richtlinie (Abschnitt 4).

\section{Anzahl Europäischer Betriebsräte in deutschen Unternehmen}

Zur Verbreitung von EBRs in der Europäischen Union gibt es eine autoritative Quelle, auf die sich nicht zuletzt auch die Europäische Kommission bei ihren Angaben bezieht: Die EBR-Datenbank des European Trade Union Institute (ETUI) (Pas/Kerckhofs 2006). Bereits im Vorfeld unseres Projektes waren wir jedoch - nicht zuletzt vom ETUI selbst - darauf hingewiesen worden, dass die Datenbank wegen der erheblichen Dynamik in dem erfassten Unternehmensfeld nicht dafür bürgen kann, den realen Ist-Zustand abzubilden. Daher wurde vom Projektteam im Frühjahr 2007 eine eigene Datenerhebung - allerdings eingegrenzt auf deutsche Unternehmen - durchgeführt, um zu ermitteln, welche deutschen Unternehmen überhaupt unter den Geltungsbereich der Richtlinie fallen und wie viele dieser Unternehmen tatsächlich einen EBR haben.

\subsection{METHODISCHE VORBEMERKUNG}

Die regionale Eingrenzung auf deutsche Unternehmen ist zwei Gründen geschuldet:

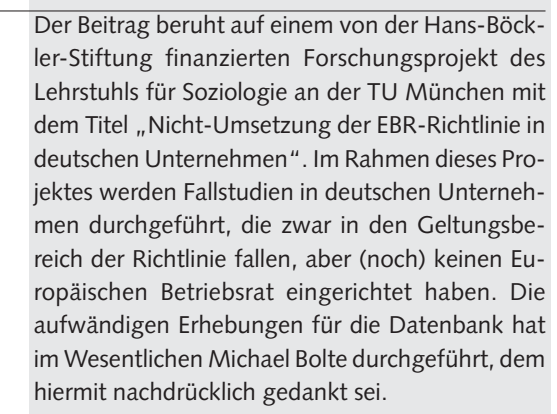
ler-Stiftung finanzierten Forschungsprojekt des Lehrstuhls für Soziologie an der TU München mit dem Titel "Nicht-Umsetzung der EBR-Richtlinie in deutschen Unternehmen ". Im Rahmen dieses Projektes werden Fallstudien in deutschen Unternehmen durchgeführt, die zwar in den Geltungsbereich der Richtlinie fallen, aber (noch) keinen Europäischen Betriebsrat eingerichtet haben. Die aufwändigen Erhebungen für die Datenbank hat im Wesentlichen Michael Bolte durchgeführt, dem hiermit nachdrücklich gedankt sei.

Stefan Lücking, Dr., wissenschaftlicher Mitarbeiter, Lehrstuhl für Soziologie, TU München. Arbeitsschwerpunkte: Europäische Arbeitsbeziehungen, prekäre Interessenvertretung, Soziologie der Wissensarbeit.

e-mail: stefan.luecking@wi.tum.de Rainer Trinczek, Prof. Dr., Lehrstuhl für Soziologie, TU München. Arbeitsschwerpunkte: Industrielle Beziehungen, Arbeitszeitforschung und Managementsoziologie. e-mail: rainer.trinczek@wi.tum.de Michael Whittall, Dr., wissenschaftlicher Mitarbeiter, Lehrstuhl für Soziologie, TU München. Arbeitsschwerpunkte: HRM, Europäische industrielle Beziehungen, Industriesoziologie.

e-mail: michael.whittall@wi.tum.de 
Zum einen zeigt die Auswertung der ETUIDatenbank, dass in deutschen Unternehmen vergleichsweise wenige EBRs bestehen. Besonders die Tatsache, dass sogar die als eher mitbestimmungsskeptisch geltenden US-amerikanischen Konzerne, die unter die Richtlinie fallen, einen relativ höheren Anteil EBRs aufweisen, wirft die Frage nach dem Verhältnis deutscher Unternehmen (und Interessenvertreter) zu der Institution EBR auf. Zum anderen ist die Eingrenzung auf deutsche Unternehmen forschungspragmatischen Gründen geschuldet und zielt darauf ab, sich auf eine überschaubare und bearbeitbare Teilmenge der existierenden EBRs zu beschränken. So zählt die 2006 veröffentlichte Fassung der EBR-Datenbank des Europäischen Gewerkschaftsinstituts (Pas/Kerckhofs 2006) 450 Unternehmen, die unseren Kriterien entsprechen. Die Einbeziehung der außereuropäischen Konzerne mit europäischem Hauptsitz in Deutschland oder gar der "gemeinschaftsweit operierenden" Unternehmen und Unternehmensgruppen, die in Deutschland aktiv sind, würde den Umfang der Untersuchung zu stark erhöhen, bis hin zu einer annähernden Vollerhebung.

Welche Unternehmen in den Geltungsbereich der Richtlinie fallen, ist durch die Definition des "gemeinschaftsweit operierenden Unternehmens" (Art. 2 Abs. 1a) bzw. der „gemeinschaftsweit operierenden Unternehmensgruppe“ (Art. 2 Abs. 1b) bestimmt. Bezugsgröße für die Richtlinie ist der Europäische Wirtschaftsraum (EWR), dem neben den Mitgliedsländern der EU Island, Liechtenstein und Norwegen angehören. Entscheidend sind die Schwellenwerte von mehr als 1000 Beschäftigten im EWR insgesamt und mehr als 150 Beschäftigten in je zwei Mitgliedsländern des EWR.

Darüber hinaus kommen nur selbstständige Unternehmen bzw. Unternehmensgruppen infrage, die nicht durch ein anderes Unternehmen beherrscht werden. Denn nach Art. 1 Abs. 3 der Richtlinie bzw. $\$ 7$ des Europäischen Betriebsräte-Gesetzes (EBRG), mittels dessen die Richtlinie in Deutschland in nationales Recht umgesetzt wurde, wird der EBR auch dann auf der Ebene des herrschenden Unternehmens eingerichtet, wenn einzelne Teile des Konzerns schon von sich aus die Schwellenwerte überschreiten - es sei denn, das besondere Verhandlungsgremium entscheidet sich bewusst für die Einrichtung von mehreren EBRs für die einzelnen Sparten. Aber auch in diesen Fällen wird die EBR-Verein- barung auf der Ebene des herrschenden Unternehmens geschlossen. Zudem geht die Definition des herrschenden Unternehmens in Art. 3 der Richtlinie bzw. \$6 EBRG weiter als die Konzerndefinition des deutschen Mitbestimmungsrechtes (Blanke 2006, S. 258f.). So reicht schon die Mehrheitsbeteiligung an einem anderen Unternehmen, um von einem beherrschenden Einfluss auszugehen.

Aus diesen Gründen war im Rahmen unserer Erhebung davon auszugehen, dass es nicht notwendig ist, alle Unternehmen und Unternehmensgruppen zu erfassen, die die Schwellenwerte aus Art. 2 der Richtlinie überschreiten, sondern nur diejenigen, die keinem beherrschenden Einfluss durch ein anderes Unternehmen unterliegen. Die Datenerhebung hat allerdings gezeigt, dass diese Kriterien, nach denen sich deutsche Unternehmen im Geltungsbereich der EBR-Richtlinie eindeutig bestimmen ließen, für die empirische Forschung in vielen Fällen unzureichend sind:

(1) Ein Problem stellen die Schwellenwerte für die Beschäftigtenzahl dar. Denn die für die Geltung der EBR-Richtlinie maßgebliche Anzahl der Beschäftigten im Europäischen Wirtschaftsraum (EWR) ist in der Regel nicht direkt verfügbar, da der EWR in aller Regel kein relevanter Bezugspunkt der Personalberichterstattung ist.

(2) Ein weiteres Problem ergibt sich aus der Definition des herrschenden Unternehmens. Bei Aktiengesellschaften lässt sich über die Aktienanteile theoretisch eindeutig bestimmen, ob ein Unternehmen durch ein anderes beherrscht wird. Da aber auch indirekte Beteiligungen zählen, kann es selbst in diesem Fall schwierig sein, einen beherrschenden Einfluss zu identifizieren. Bei Unternehmensgruppen, die aus Unternehmen bestehen, die nicht an der Börse gehandelt werden und die nicht durch Mehrheitsbeteiligungen verbunden sind, gilt das Kriterium der einheitlichen Leitung, das sich von außen aber nur schwer überprüfen lässt. Für die Datenerhebung sind wir dann von einem beherrschenden Einfluss ausgegangen, wenn sich das Unternehmen in seiner öffentlichen Selbstdarstellung (d.h. im Internet und in Geschäftsberichten) als Hauptsitz einer größeren Unternehmensgruppe darstellt.

(3) Die Datenerhebung hat zudem ergeben, dass in einigen Unternehmensgruppen nicht nur mehrere EBRs existieren, sondern auch mehrere unabhängige Vereinbarungen zur Einrichtung eines EBR. Die Existenz mehrerer EBRs ist in den seltensten Fällen das Ergebnis einer bewussten Entscheidung, sondern ein historisches Relikt oder der Tatsache geschuldet, dass Konzernteile, an denen das herrschende Unternehmen eine Aktienmehrheit hält, weiterhin als selbstständige Unternehmen betrachtet werden. Die entscheidende Frage dabei ist, wie Konzerne gezählt werden, die mehrere EBRs haben. Zählt man jeden Konzernteil einzeln, der über einen eigenen EBR verfügt, ist der „Deckungsgrad“ höher, als wenn ein Konzern nur einmal zählt - unabhängig davon, wie viele EBRs in ihm bestehen. ${ }^{2}$

(4) Eine letzte Schwierigkeit ergibt sich aus dem steten Wandel der Unternehmenslandschaft. Selbst in der kurzen Zeit der Datenerhebung im Frühjahr 2007 hat es eine Reihe von Fusionen, Übernahmen und Veräußerungen gegeben. Seither sind noch einige, hier nicht erhobene Veränderungen hinzugekommen.

Die Komplikationen bei der Datenerhebung führen zu einem entscheidenden Ergebnis: Es ist nicht nur „schwierig“, den Anteil der Unternehmen mit EBR an allen Unternehmen zu berechnen, die in den Geltungsbereich der Richtlinie fallen; es ist in einem streng methodischen Sinne letztlich sogar unmöglich, einen „Deckungsgrad“ zu bestimmen, weil die Grundgesamtheit auf der Basis der aktuellen Bestimmungen der EBR-Richtlinie sowie des EBRG nicht eindeutig zu definieren ist.

\subsection{DEUTSCHE UNTERNEHMEN IM GELTUNGSBEREICH DER EBR-RICHTLINIE}

Die Ergebnisse unserer Datenerhebung (TUM-Datenbank) zeigen vordergründig keine größeren Abweichungen von den $\mathrm{Da}$ ten der ETUI-Datenbank. Von den Unternehmen, die aufgrund der vorliegenden

\footnotetext{
2 Das vorliegende Projekt und die EBR-Datenbank des Europäischen Gewerkschaftsinstituts (ETUI) basieren auf einem Mittelweg: Es werden die Konzernteile einzeln gezählt, für die es eine eigene Vereinbarung zur Einrichtung eines Euro-Betriebsrates gibt oder für die im Unterschied zu anderen Teilen des Konzerns kein Euro-Betriebsrat existiert. Konzerne, die über eine einheitliche EBR-Vereinbarung mehrere Sparten-Betriebsräte eingerichtet haben, werden dagegen als Einheit gewertet.
} 
Daten in den Geltungsbereich der Richtlinie fallen, verfügen weniger als $30 \%$ tatsächlich über einen EBR (131 bzw. 28,3 \% in der TUM-Datenbank, 123 bzw. 27,3\% in der EBR-Datenbank des ETUI).

Diese Ähnlichkeit bei der quantitativen Verbreitung von EBRs täuscht jedoch über die teilweise erheblichen Veränderungen hinweg, die sich seit der Erhebung der EBRDatenbank des ETUI ergeben haben. Von den deutschen Unternehmen, die der ETUI-Datenbank zufolge im Sommer 2005 von der EBR-Richtlinie erfasst waren, haben 57 durch Übernahmen oder Fusionen ihre Unabhängigkeit verloren, sieben sind in Insolvenz gegangen und weitere 53 erfüllen nach unserer Einschätzungen die Voraussetzungen der EBR-Richtlinie nicht (mehr). Dagegen haben wir 152 Datensätze neu eingetragen. Und 23 Unternehmen, die dem ETUI zufolge nicht in den Geltungsbereich der Richtlinie fallen, erfüllen die Voraussetzungen inzwischen. Gleichzeitig sind von den 123 deutschen Unternehmen, die der ETUI-Datenbank zufolge einen EBR hatten, inzwischen zwei in Insolvenz gegangen und 17 weitere durch ein anderes Unternehmen übernommen worden, sodass sie in unserer Datenbank nicht mehr als selbstständige Unternehmen geführt werden. Hinter der scheinbaren Kontinuität bei der quantitativen Verbreitung verbergen sich demnach deutliche Veränderungen.

Die weitere statistische Auswertung der Daten stößt sehr schnell an Grenzen. Bei einem Branchenvergleich ergibt sich das Problem, dass die Zahl der gemeinschaftsweiten deutschen Unternehmensgruppen in manchen Sektoren so gering ist, dass es auch unabhängig vom Problem einer eindeutigen Grundgesamtheit - wenig Sinn macht, den Anteil der Unternehmensgruppen mit EBR in Prozent zu berechnen. In einigen Sektoren ist schon der Anteil der Unternehmen, in denen es aktuell Verhandlungen über einen EBR gibt, recht hoch, obwohl es sich jeweils nur um zwei Fälle handelt. Deshalb ist in dem folgenden Schaubild, das die Branchensystematik des Europäischen Gewerkschaftsinstituts verwendet, die Fallzahl für die einzelnen Sektoren angegeben (Abbildung 2).

Für eine exakte Auswertung, wie sich die Größe einer Unternehmensgruppe (gemessen an der Anzahl der dort Beschäftigten) auf die Existenz oder Nicht-Existenz eines EBR auswirkt, sind die Angaben über Beschäftigtenzahlen zu unvollständig. Die
Zahl der Beschäftigten im Europäischen Wirtschaftsraum konnten wir nur für gut ein Drittel der Unternehmen bestimmen. Im Hinblick auf die Zahl der Beschäftigten weltweit, die wir für 442 von 463 Unternehmen erfasst haben, ergibt sich das zu erwartende Bild: Je größer eine Unternehmensgruppe, desto höher ist die Wahrscheinlichkeit, dass sie über einen EBR verfügt (Abbildung 3).

\section{Problemzonen für Europäische Betriebsräte}

Im Zuge der Datenerhebung wurde zunehmend klar, dass die Probleme bei der exakten Erfassung der quantitativen Verbreitung von Europäischen Betriebsräten (EBR) oft direkt mit Gründen zusammenhängen, die auch für die beteiligten Akteure typische Problemzonen bei der Einrichtung eines EBR darstellen. Wie die Probleme des Forschungsteams mit realen Problemen in der Unternehmenspraxis zusammenhängen, soll im Folgenden an drei Beispielen gezeigt werden: Unterneh-
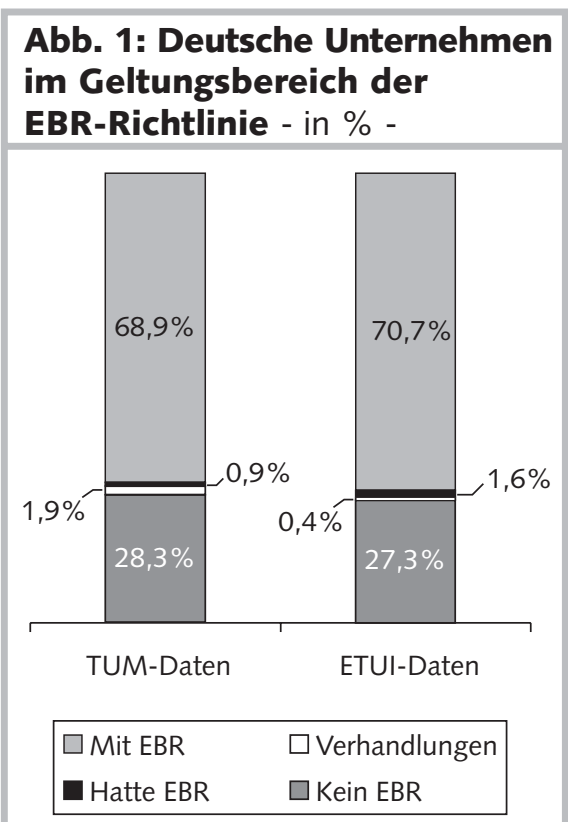

Quelle: Darstellung der Autoren.

WSI MITTEILUNGeN

mensübernahmen und -fusionen, den uneinheitlichen EBR-Strukturen in manchen Unternehmen sowie der notorischen Intransparenz von Eigentümerunternehmen.

\section{Abb. 2: Euro-Betriebsräte in deutschen Unternehmen nach Branchen (2007)}

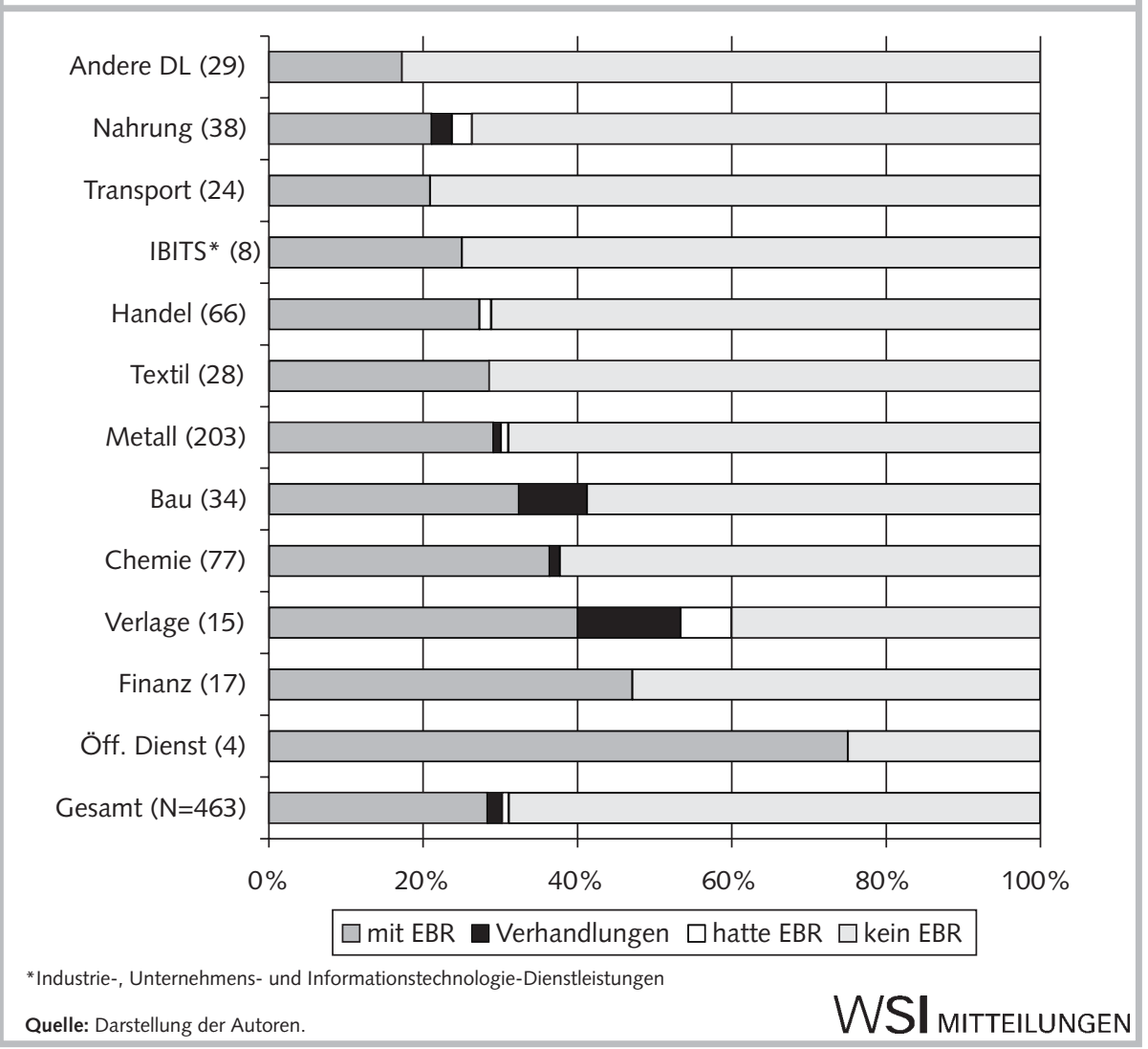




\section{Abb. 3: Euro-Betriebsräte in deutschen Unternehmen nach Beschäftigtenzahl - in \% -}

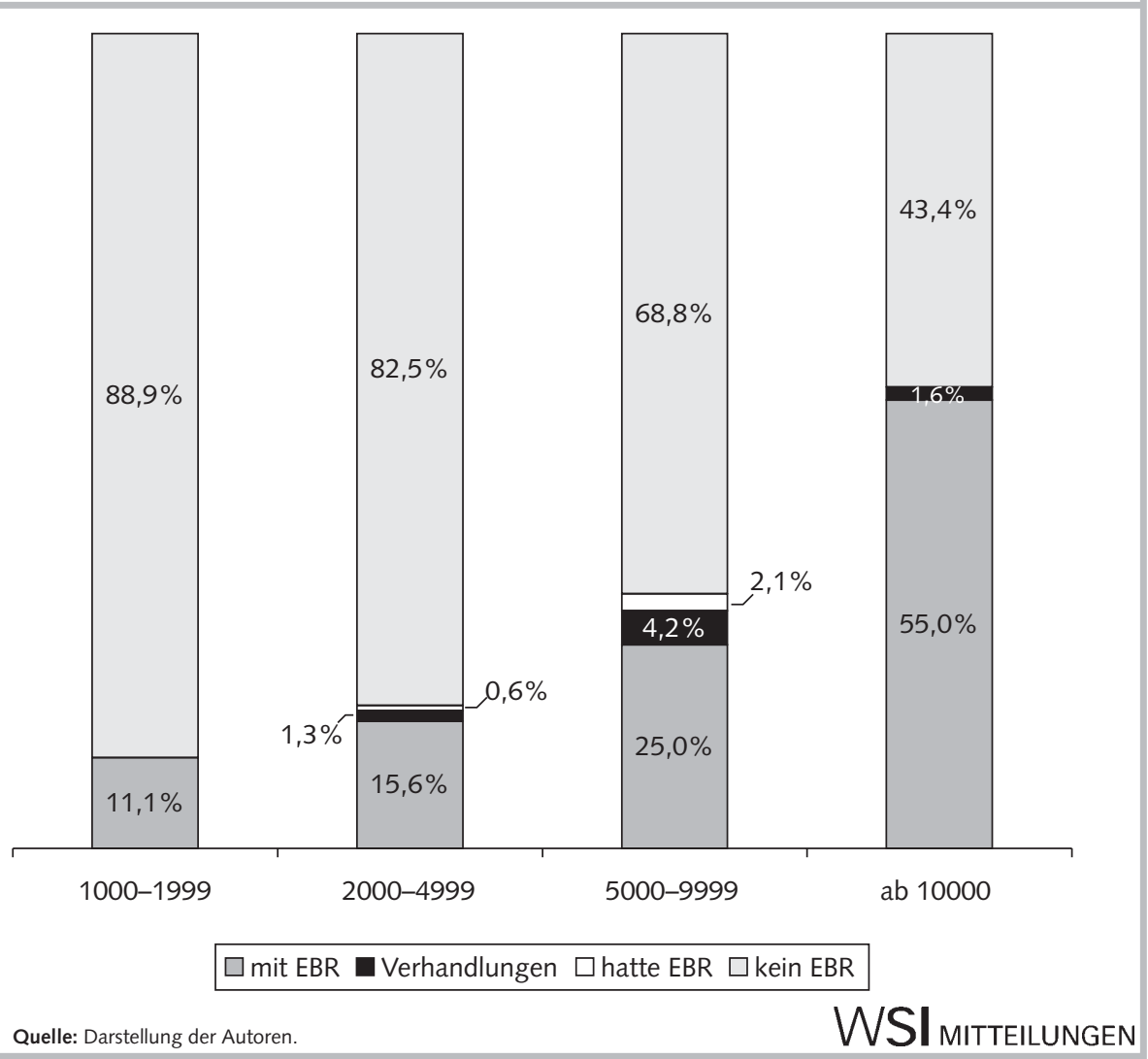

\subsection{UNTERNEHMENSAKQUISITIONEN UND FUSIONEN}

Ein großes Problem für die Datenerfassung ist die stetige Veränderung der Datenbasis aufgrund des permanenten Wandels der Unternehmenslandschaft in Europa: Fusionen, Übernahmen, Unternehmensverkäufe, Insolvenzen usw. schaffen ständig neue Kontextbedingungen für die Gründung neuer, aber auch für die Arbeit existierender EBRs.

Bei unserer Datenerhebung sind wir auf viele Unternehmen gestoßen, die inzwischen aufgrund von Fusionen oder Übernahmen ihre Selbstständigkeit verloren haben. Unter diesen Fusionen und Übernahmen wurden 42 Fälle identifiziert, in denen mindestens ein EBR involviert war, der aufgrund der veränderten Unternehmensstrukturen ebenfalls neu geordnet werden musste. Angesichts der Tatsache, dass es sich dabei um $32 \%$ aller deutschen Unternehmensgruppen mit EBR handelt, die in unserer Datenbank erfasst sind, ist das ein sehr hoher Wert. Zudem wurden nur die Fälle berücksichtigt, bei denen ein gemeinschaftsweites Unternehmen durch ein anderes übernommen wurde.
Dies ist insbesondere deswegen so kritisch, weil die existierende Richtlinie zu den EBRs keine Regelungen vorsieht, wie zu verfahren ist, wenn eine bestehende EBRStruktur infolge einer solchen Restrukturierung neu geordnet werden muss oder wenn zwei Unternehmen mit eigenem EBR fusionieren. Streng genommen verlieren bei einer Fusion alte EBR-Gremien ihre Zuständigkeit, und es müsste in diesen Fällen ein besonderes Verhandlungsgremium gebildet werden, um eine neue EBR-Vereinbarung auszuhandeln. Dies ist misslich, da dadurch ja gerade in der entscheidenden frühen Post-Merger-Phase der EBR als kollektive Interessenvertretung der Beschäftigten sowie als Gesprächs- und Verhandlungspartner ausfällt, obwohl gerade solche Konstellationen eigentlich den Kernbereich der Aufgaben eines EBR darstellen (Carley/Hall 2006).

Die Europäische Kommission schlägt für diese Fälle die Einführung einer Anpassungsklausel vor. Demnach muss jede neue EBR-Vereinbarung „die Modalitäten ihrer Anpassung und Kündigung sowie ihrer Neuaushandlung" nennen. Für die Fälle, in denen eine ältere Vereinbarung vorliegt, die noch keine entsprechende Bestimmung enthält, oder mehrere Vereinbarungen mit unterschiedlichen Bestimmungen vorliegen, sieht der Vorschlag der Kommission eine Verhandlungspflicht sowie einen Übergangsmechanismus vor, der gewährleistet, das bestehende EBR-Gremien für die Dauer der Neuverhandlung handlungsfähig bleiben (Europäische Kommission 2008, S. 7f.).

Die in unserer Datenbank identifizierten 42 Fälle von Fusionen mit EBR können als Anschauungsmaterial dafür dienen, wie dieses Problem in der Praxis bislang gelöst wird und wie eine sinnvolle Anpassungsklausel aussehen könnte. Die Datenerhebung war größtenteils auf öffentlich zugängliche Informationen beschränkt. Nur in wenigen Fällen haben wir uns in Telefongesprächen mit den Betriebsräten vergewissert, ob ein vor der Fusion bestehender EBR noch existiert. Schon aus diesen wenigen Informationen ergeben sich aufschlussreiche Erkenntnisse.

Im Idealfall gibt es für die Neuregelung der EBR-Struktur nach einer Fusion ein Post-Merger-Agreement. Normalerweise regelt eine solche Vereinbarung die Fusion zweier bereits bestehender EBRs. In einigen Fällen kommt es aber auch zu anderen Lösungen. Im Anschluss an die Übernahme der Felten \& Guilleaume Kabelwerke durch die dänische NKT Group wurde nach der Fusion eine neue EBR-Struktur vereinbart. Diese sieht vor, dass der EBR der Felten \& Guilleaume Kabelwerke bestehen bleibt und die Zuständigkeit für die gesamte Konzernsparte NKT Cables übernimmt. Zusätzlich wird ein gemeinsamer EBR auf Konzernebene eingerichtet. Die zweite Konzernsparte Nilfisk-Advance Group erhält ebenfalls einen eigenen EBR. Im Fall der Übernahme der Rheinhold \& Mahla GmbH durch Bilfinger Berger wurde das neu hinzugekommene Unternehmen nicht in den bestehenden EBR aufgenommen, sondern es wurde nach $\mathrm{Ab}$ schluss der Restrukturierung ein eigener EBR für die neu entstandene Konzernsparte Bilfinger Berger Industrial Services vereinbart.

$\mathrm{Zu}$ beachten ist auch, dass die Übernahme einer Aktienmehrheit nicht unbedingt zu einer organisatorischen Integration in den Konzern führen muss. Im Fall der Übernahme der Beiersdorf AG durch die Tchibo Holding wurde die Holding schließlich in maxingvest ag umbenannt, um die Selbstständigkeit der beiden Konzernsparten Tchibo und Beiersdorf zu ver- 
deutlichen. Außerdem ist es nicht selten, dass ein Unternehmen übernommen und später wieder verkauft wird. In solchen Fällen wäre es sinnlos, im Anschluss an die Übernahme den EBR anzupassen.

Ein besonders vertracktes Beispiel ist die SKW Trostberg AG, die 2001 zusammen mit der Degussa-Hüls zur Degussa AG fusionierte. Bereits 2004 wurde die StahlMetallurgie-Sparte an die Beteiligungsgesellschaft ARQUES Industries AG verkauft, die sich darauf spezialisiert hat, Großkonzernen bei deren Konzernabspaltungen zu helfen. Diese brachte 2006 die SKW StahlMetallurgie Holding AG an die Börse und verkaufte 2007 die restlichen Anteile, sodass das Unternehmen aktuell wieder selbstständig ist. Der 1995 gegründete EBR der SKW Trostberg hat diese Veränderungen in den Eigentumsverhältnissen nicht überstanden.

Die in unserer Datenbank identifizierbaren Fälle zeigen, dass die Vorschläge der Kommission wohl nicht ausreichen dürften, insbesondere weil sie sich nur auf neu abzuschließende EBR-Vereinbarungen beziehen. Sinnvoll wäre der Beschluss von Standardprozeduren, die in allen Fällen greifen, in denen keine eindeutige Anpassungsklausel vorliegt. Vor allem für die Fälle, in denen beide Unternehmen bereits über einen EBR verfügen, sollte es nicht nötig sein, ein neues besonderes Verhandlungsgremium einzurichten. Stattdessen könnte die neue Vereinbarung durch die bestehenden EBRs ausgehandelt werden. Außerdem sollte die Möglichkeit eingeräumt werden, bestehende EBR-Strukturen beizubehalten. Den Übergangsregeln für die Zeit bis zur Neuaushandlung der EBRStruktur sollte besondere Aufmerksamkeit gewidmet werden. Denn gerade für die Zeit nach einer Fusion sollte ein bestehender EBR handlungsfähig sein, weil Restrukturierungsmaßnahmen erst im Anschluss an die Fusion erfolgen.

\subsection{UNEINHEITLICHE EUROPÄISCHE BETRIEBSRATSSTRUKTUREN}

Unternehmensgruppen, in denen mehrere EBR-Vereinbarungen existieren, stellen nicht nur für die statistische Auswertung der Daten ein Problem dar, sondern auch für die Umsetzung der EBR-Richtlinie insbesondere dann, wenn innerhalb eines Konzerns Sparten mit EBR und solche ohne EBR existieren. In den meisten Fällen dürften die Gründe für diese uneinheitli- chen EBR-Strukturen historischer Art sein. In einigen Fällen wurde ein Unternehmen mit EBR von einem Unternehmen ohne EBR übernommen. Eigentlich müsste in solchen Fällen ein neuer EBR auf der Ebene der zentralen Leitung eingerichtet werden. Wenn sich die Verhandlungen dazu aber als schwierig erweisen, scheint in einigen Fällen die Lösung darin zu bestehen, den alten EBR bestehen zu lassen - zumindest solange die Verhandlungen über einen neuen EBR andauern. Ein Beispiel dafür ist nach unseren Erkenntnissen die Franz-Haniel-Gruppe. In anderen Fällen wurden neu hinzugekommene Konzernteile nicht in die bestehenden EBR-Strukturen integriert.

In manchen Fällen wurden getrennte EBR-Strukturen nach einer Fusion durch ein Post-Merger-Agreement bewusst festgeschrieben. Dies war nach unseren Kenntnissen etwa bei Bilfinger Berger und der dänischen NKT Group der Fall. In anderen Fällen wurden von vornherein für einzelne Konzernteile eigenständige EBR-Vereinbarungen abgeschlossen, so etwa für Gruner + Jahr und die RTL Group innerhalb des Bertelsmann-Konzerns. In diesen Fällen sehen wir den Grund für die uneinheitlichen EBR-Strukturen in den klar getrennten Unternehmensstrukturen. Die betroffenen Konzernteile sind zwar aufgrund der Besitzverhältnisse Teil des Konzerns, werden von den betrieblichen Akteuren aber dennoch als eigenständige Unternehmensgruppen wahrgenommen und sind organisatorisch klar genug getrennt, sodass ein eigener EBR sinnvoll erscheint.

In den Fällen, in denen eine Unternehmensgruppe mit EBR durch ein Unternehmen ohne EBR beherrscht wird, liegt in unserer Interpretation ein weiterer bedeutsamer Grund vor: unterschiedliche Kräfteverhältnisse bzw. Konkurrenz zwischen verschiedenen Gewerkschaftsorganisationen oder Standorten innerhalb des Konzerns. Ein aus der Presse bekanntes Beispiel ist die Münchener Rück. Hier ist es erst im Sommer 2007 nach zähen Verhandlungen gelungen, einen gemeinsamen Konzernbetriebsrat mit der (von der Anzahl der Beschäftigten größeren) ERGO-Gruppe zu bilden, weil die Betriebsräte der ERGO mehrheitlich bei ver.di organisiert sind, der Betriebsrat der Münchener Rück dagegen aus Nicht-Organisierten und DHV ${ }^{3}$-Mitgliedern besteht. Der Betriebsrat der Münchener Rück hatte deshalb wenig Interesse an einem mehrheitlich bei ver.di organi- sierten Konzernbetriebsrat und genauso wenig an einem gemeinsamen EBR.

Die Frage, wie mit solchen uneinheitlichen EBR-Strukturen in Zukunft umgegangen werden soll bzw. wie solche Strukturen vermieden werden können, wird in den aktuellen Reformvorschlägen der Europäischen Kommission allerdings nicht thematisiert.

\subsection{EIGENTÜMERUNTERNEHMEN ALS DEUTSCHER SONDERFALL}

Eigentümerunternehmen und Unternehmensgruppen, die nicht an der Börse notiert sind und deshalb nur eingeschränkten Veröffentlichungspflichten unterliegen, sind eine Besonderheit der deutschen Unternehmenslandschaft. Laut Institut für Mittelstandsforschung ist die Einheit von Eigentum und Leitung nicht nur der dominierende Unternehmenstyp in der deutschen Unternehmenslandschaft, sondern diese Unternehmen bilden auch die tragende Säule für Wachstum und Beschäftigung (Wallau et al. 2007). Die Bedeutung von Eigentümerunternehmen wird auch in unserer EBR-Datenbank nachgewiesen. Sie stellen mit 270 von 468 (58 \%) die Mehrheit der deutschen Unternehmen, die in den Geltungsbereich der Richtlinie fallen. Im Hinblick auf die Existenz eines EBR ergibt sich ein ganz anderes Bild: In den Eigentümerunternehmen sind nur $38 \%$ aller EBRs installiert.

Die Relevanz dieses Unternehmenstypus spiegelt sich auch im qualitativen Teil unserer Analyse. Drei von insgesamt sechs Fallstudien sind Kommanditgesellschaften und bieten interessante empirische Daten. Sie verweisen auf mehrere Faktoren, die es erschweren, einen EBR zu gründen. Häufig ist bereits unklar, ob ein Unternehmen in den Geltungsbereich der Richtlinie fällt oder nicht. Drei Aspekte spielen hierbei eine große Rolle: die reduzierte Informationspflicht von Eigentümerunternehmen, die mitunter ablehnende Einstellung der Eigentümer gegenüber der Mitbestimmung und die teilweise undurchsichtigen Unternehmensstrukturen. Auf diese drei Aspekte wird im Folgenden kurz eingegangen.

3 DHV - Die Berufsgewerkschaft ist die Organisation der kaufmännischen und verwaltenden Berufe im Christlichen Gewerkschaftsbund Deutschlands (CGB). 


\section{REDUZIERTE INFORMATIONSPFLICHT}

Während Aktiengesellschaften definierte Veröffentlichungspflichten haben, trifft dies auf Eigentümerunternehmen (sofern sie eine andere Gesellschaftsform gewählt haben) nur in eingeschränktem Maße zu. So kommt es, dass beispielsweise viele Eigentümerunternehmen keine Beschäftigtenzahlen veröffentlichen. Deshalb lässt sich von außen nicht ohne Weiteres feststellen, ob die Schwellenwerte für die Einrichtung eines EBR überschritten werden. Außerdem zeigt sich das Management offensichtlich nicht immer bereit, solche Informationen den internen Arbeitnehmervertretern mitzuteilen. Wie mehrere Rechtsfälle zeigen, ist die Frage, ob die Schwellenwerte überschritten sind, ein altes Problem, vor allem ein deutsches. Es gibt drei Fälle, Bofrost (2001), Kühne \& Nagel (2004) und ADS Anker (2004), alle drei deutsche Arbeitgeber, die vor den Europäischen Gerichtshof gekommen sind, weil sie sich geweigert haben, entsprechende Informationen zu erteilen. Laut Europäischem Gerichtshof verstößt eine solche Weigerung gegen die EBR-Richtlinie.

Die Betriebsräte unserer Fallstudien wussten zum großen Teil nur, dass ihre Firmen in bestimmten EU-Ländern tätig waren. Nur in einem Fall waren die aktuellen Beschäftigtenzahlen bekannt. Selbst wenn das Management bereit ist, solche Information zu teilen, besteht ein weiteres Problem für Arbeitnehmervertreter darin, in Erfahrung zu bringen, ob an den anderen europäischen Standorten überhaupt schon Strukturen einer Arbeitnehmervertretung vorhanden sind, mit denen man einen EBR etablieren könnte.

Weiterhin scheint es von der jeweiligen betriebspolitischen Konstellation abzuhängen, ob ein Betriebsrat bereit ist, seine Informationsrechte einzuklagen; faktisch wird dies eher selten getan. Nur in einem Fall hat ein Betriebsrat, und hier mit Hilfe der entsprechenden Gewerkschaft, versucht zu prüfen, ob die Firma unter die Richtlinie fällt, und zusätzlich, ob entsprechende Vertretungsstrukturen auf Arbeitsnehmerseite an den anderen Standorten existierten. Dieser Schritt erfolgte, nachdem der Vorstand entschieden hatte, bestimmte Produktionsteile ins Ausland zu verlagern - eine Entscheidung, die Arbeitsplätze in Deutschland gefährdete.

\section{MITBESTIMMUNGSABLEHNUNG}

Eigentümerunternehmen sind häufig von einer ablehnenden Haltung gegenüber dem Mitbestimmungsgedanken im Allgemeinen und rechtlich verankerten Institutionen der Arbeitnehmervertretung im Besonderen geprägt. Wie eine Studie über Familienunternehmen in Europa (Stiftung Familienunternehmen 2006a) zeigt, ist die Mitbestimmung bei Eigentümerunternehmen höchst umstritten, bis hin zu der Forderung ,auch die heilige Kuh der Mitbestimmung als Relikt aus „Klassenkampfzeiten " zu überprüfen " (Stiftung Familienunternehmen 2006b).

Bei den „Problemfällen“ handelt es sich insbesondere um Unternehmensgruppen, die trotz ihrer Größe schon auf nationaler Ebene keine funktionierende Betriebsratsstruktur aufweisen. Ein großer Teil der Unternehmensgruppen, zu denen überhaupt keine Beschäftigtenzahlen zu ermitteln waren, gehört dieser Gruppe an. Eine restriktive Informationspolitik ist deshalb bereits ein Hinweis darauf, dass eine Unternehmensgruppe ein schwieriges Terrain für die Gründung eines EBR darstellt. Hierfür finden sich auch in unseren Interviews diverse Hinweise. So sprechen zwei interviewten Personalleitern zufolge drei Faktoren gegen die Gründung eines EBR: erstens die hohen Kosten eines EBR, zweitens mehr Bürokratie und drittens zu viel Transparenz. Vor allem der letzte Faktor, Transparenz, scheint ein großes Problem für das Management zu sein, da es befürchtet, dass Arbeitnehmer den EBR dazu nutzen, ihre Arbeitsund Entlohnungsbedingungen zu vergleichen und dadurch eine Anspruchsspirale in Gang gesetzt wird.

In einzelnen Fällen wird die restriktive Informationspolitik zusätzlich durch eine besonders undurchsichtige Konzernstruktur unterstützt. Bei einzelnen Unternehmensgruppen waren mehrstündige Recherchen erforderlich, um festzustellen, welche Unternehmen zur Gruppe gehören und welches Unternehmen in dem Konzerngeflecht die Funktion des herrschenden Unternehmens hat. Ein besonders extremes Beispiel stellt die Arend Oetker Holding dar, zu der neben den Schwartauer Werken ein ganzes Netzwerk aus Unternehmen gehört, die in den unterschiedlichsten Branchen tätig sind. An ihrer Spitze steht mit der in Bad Schwartau ansässigen $\mathrm{AOH}$ Nahrungsmittel GmbH \& Co. KG eine reine Holdinggesellschaft, die nur indirekt über zwei Schweizer Aktiengesellschaften, die FIM AG und die Hero AG, mit den eigentlichen Produktionsstätten verbunden ist. Die restriktive Informationspolitik und die undurchsichtige Konzernstruktur dienen hier offensichtlich auch dazu, die Etablierung einer konzernweiten Interessenvertretung zu verhindern.

\section{UNTERNEHMENSSTRUKTUREN}

Der Fall der Arend Oetker Holding verweist darauf, dass die Firmenstruktur die Gründung eines EBR erschweren kann. Es handelt sich hier um die Frage, in welcher Beziehung die verschiedenen Tochtergesellschaften zum Mutterkonzern stehen, der sogenannte Unabhängigkeitsfaktor. Wie es im Hinblick auf den Gesamtbetriebsrat, den Konzernbetriebsrat und den Aufsichtsrat hinlänglich bekannt ist, können Firmen ihre Besitzstrukturen gezielt gestalten, um bestehende Mitbestimmungsrichtlinien zu umgehen.

In Bezug auf die EBR-Richtlinie bedeutet dies, dass Firmen nach Wegen suchen, die Definitionen europaweit operierender Unternehmen oder Unternehmensgruppen nach Art. 2 Abs. 1 zu umgehen, indem sie den Konzern in rechtlich unabhängige Unternehmensgruppen aufteilen, die jeweils unter den Schwellenwerten liegen. In einer unserer drei Fallstudien hat die Firma eine Holding in der Schweiz errichtet, um das zu ermöglichen. Mindestens auf dem Papier könnte eine Reihe von ausländischen Tochtergesellschaften als unabhängig von der Zentrale betrachtet werden. In einem anderen Fall hat das Management eine Struktur aufgebaut, bei der die einzelnen Firmen im Besitz verschiedener Familienmitglieder sind, die formalrechtlich unabhängig voneinander agieren, faktisch jedoch gemeinsam geführt werden.

Laut EBRG hängt der Status einer Tochtergesellschaft als Teil einer Unternehmensgruppe davon ab, ob ein herrschendes Unternehmen „unmittelbar oder mittelbar einen beherrschenden Einfluss auf ein anderes Unternehmen derselben Gruppe (abhängiges Unternehmen) ausüben kann" ( $\$ 6$ Abs. 1 EBRG). Um herauszufinden, ob es ein herrschendes Unternehmen gibt, muss dem deutschen EBRG zufolge mindestens einer von drei Faktoren vorliegen ( $\$ 6$ Abs. 2 EBRG, vgl. Blanke 2006, S. 259-262). Es muss 
- mehr als die Hälfte der Mitglieder des Verwaltungs-, Leitungs- oder Aufsichtsorgans des anderen Unternehmens bestellen können oder

- über der Mehrheit der mit den Anteilen am anderen Unternehmen verbundenen Stimmrechte verfügen oder

- die Mehrheit des gezeichneten Kapitals dieses Unternehmens besitzen.

In allen drei Fallstudien stellte das Management die Existenz eines herrschenden Unternehmens infrage. Im Zuge der Empirie - vor allem aufgrund der Interviews mit dem Management und Arbeitnehmervertretern der ausländischen Tochtergesellschaften - konnte jedoch festgestellt werden, dass die Zentrale in aller Regel eine beherrschende Rolle spielt. In einem Fall berichtete ein Interviewpartner, dass der Besitzer mehrmals im Jahr die ausländischen Tochtergesellschaften besucht. In weiteren Interviews in derselben Firma ergab sich, dass in den letzten Jahren die Zentrale eine Controlling-Abteilung gegründet hatte, um die ausländischen Tochtergesellschaften besser zu kontrollieren. In einem anderen Fall ist die Zentrale verantwortlich für die Einstellung von Führungskräften im Ausland.

Die Möglichkeiten zur Gründung eines EBR scheinen also in Eigentümerbetrieben insbesondere davon abzuhängen, ob das Management bereit ist, die notwendigen Informationen (zur Struktur der Unternehmensgruppe, zur Anzahl der Beschäftigten an den ausländischen Standorten etc.) ohne größere Verschleierungsversuche weiterzugeben. So gesehen spielen die Führungskräfte eine Gate Keeper-Rolle.

Das Arbeitspapier der Europäischen Kommission greift die für diese Problemzone relevanten Fragen bezüglich Schwellenüberschreitung und herrschendem Unternehmen als Transparenzproblem auf. Abschnitt 2.2 dieses Papiers legt fest, „dass sämtliche Informationen, die für die Aufnahme der Verhandlungen über die Bildung eines Europäischen Betriebsrats unerlässlich sind, von den Unternehmensleitungen der betreffenden Unternehmen in den Mitgliedstaaten den Arbeitnehmervertretern zur Verfügung gestellt werden müssen" (Europäische Kommission 2007, S. 8). Dabei stützt die Europäische Kommission ihr Papier explizit auf die Entscheidungen des Europäischen Gerichtshofs in Bezug auf die Fälle Bofrost, Kühne \& Nagel und ADS Anker.

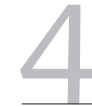 Schlussfolgerungen}

Die Datenerhebung zu den deutschen Unternehmen im Geltungsbereich der EBRRichtlinie hat eine Reihe aufschlussreicher Ergebnisse erbracht. Erstens hat sich herausgestellt, dass eine statistische Auswertung in Form von „Deckungsgraden“ problematisch ist, weil die Anzahl der Unternehmen, die unter die Richtlinie fallen, nicht eindeutig bestimmbar ist. Eine Lösung wäre, den „Deckungsgrad“ mit Bezug auf die Zahl der betroffenen Beschäftigten zu bestimmen. Dazu reichen die Daten aber bei Weitem nicht aus, weil sich für viele Unternehmen die Anzahl der Beschäftigten im Europäischen Wirtschaftsraum höchstens schätzen lässt. ${ }^{4}$

Das bedeutet jedoch nicht, dass die Erhebung von Daten sinnlos wäre. Denn die Daten bieten, zweitens, wichtige Informationen über die Problemzonen für die Einrichtung eines Europäischen Betriebsrats (EBR). Das haben wir an drei Beispielen erläutert: Fusionen, in denen EBRs involviert waren, Konzerne mit uneinheitlichen EBRStrukturen und Eigentümerunternehmen, die in Bezug auf Beschäftigtenzahl und Unternehmensstruktur intransparent sind.

Auf zwei dieser Problemzonen geht die Europäische Kommission in ihren Vorschlägen ein. Zur Lösung des Problems von Fusionen, Übernahmen, Unternehmensverkäufen und anderen grundlegenden Veränderungen der Unternehmensstruktur, die eine Neuordnung der EBR-Struktur erfordern, schlägt sie eine Anpassungsklausel und, sofern diese fehlt oder nicht eindeutig ist, eine Neuverhandlungspflicht und einen Übergangsmechanismus vor. Unsere Datenerhebung belegt nicht nur die zahlenmäßige Bedeutung dieses Problems, sondern zeigt auch, dass zusätzlich zu den Vorschlägen der Kommission eine Regelung für ein Standardverfahren notwendig wäre, das die Neuaushandlung der EBRStruktur vor allem in den Fällen vereinfacht, in denen zwei Unternehmensgruppen mit eigenem EBR und unterschiedlichen Anpassungsklauseln fusionieren.

Die Problemzone der Eigentümerunternehmen, die im Hinblick auf Beschäftigtenzahl und Konzernstruktur intransparent sind, greift die Europäische Kommission mit dem Vorschlag auf, die Verpflichtung, alle Informationen, die für die Frage entscheidend sind, ob eine Unternehmensgruppe in den Geltungsbereich der EBRRichtlinie fällt, explizit offenzulegen. $\mathrm{Ob}$ das ausreicht, kann jedoch bezweifelt werden. Denn schließlich ergibt sich diese Veröffentlichungspflicht bereits eindeutig aus der Rechtsprechung des Europäischen Gerichtshofes. Im Konfliktfall wären die Arbeitnehmervertretungen nach wie vor gezwungen, den Rechtsweg zu beschreiten. Idealerweise sollte es ein verpflichtendes europaweites Melderegister für Unternehmen geben, die die Kriterien der EBRRichtlinie erfüllen.

Auf die dritte Problemzone, Konzerne mit uneinheitlichen EBR-Strukturen, geht das Arbeitspapier der Europäischen Kommission nicht ein, obwohl die damit verbundenen Vertretungslücken mit dem rechtlichen Grundsatz in Konflikt stehen, dass durch den EBR alle europäischen Arbeitnehmer des Konzerns vertreten sein sollen. Dass dieses Thema fehlt, liegt jedoch auch daran, dass es in der Diskussion um die Revision der EBR-Richtlinie kaum beachtet wurde. Ob und wie das Problem solcher Vertretungslücken im Rahmen der Revision der Richtlinie angegangen werden kann, setzt genauere Kenntnisse der Ursachen voraus. Dazu wären Fallstudien zu Konzernen mit uneinheitlicher EBR-Struktur erforderlich.

Auch bei den anderen Problemzonen zeigt unsere Datenerhebung, dass es noch erheblichen Forschungsbedarf gibt. So erlaubt es unsere Datenbank zwar - beispielsweise im Hinblick auf Fusionen und andere Veränderungen der Unternehmensstruktur - Fälle zu identifizieren, in denen ein EBR neu verhandelt werden musste oder überhaupt erst gegründet wurde. Für ein genaues Verständnis der Prozesse, die dabei abgelaufen sind, und der Lösungen, die entwickelt wurden, um eine völlige Neuverhandlung zu vermeiden, wären jedoch intensive Fallstudien erforderlich. Die Ergebnisse solcher Fallstudien könnten helfen, geeignete Regelungen für eine Revision der Richtlinie zu finden. Ähnlicher Forschungsbedarf besteht mit Blick auf das breit gefächerte Spektrum von Eigentümerunternehmen und deren unterschiedliche Politiken gegenüber EBRs.

\footnotetext{
4 Die Schätzungen des Europäischen Gewerkschaftsinstituts aber zeigen, dass in diesem Fall der Deckungsgrad deutlich höher liegt, als wenn er auf die Anzahl der Unternehmen bezogen wird (61 \% statt $35 \%$ für den EWR insgesamt (Kerckhofs 2006, S. 33))
} 
Altmeyer, W. (2004): Die Konzernmutter muss ihren Sitz nicht in der EU haben. Urteil des Europäischen Gerichtshofes zur Informationspflicht des Arbeitgebers bei Gründung eines Europäischen Betriebsrats, in: Arbeitsrecht im Betrieb 4, S. 204-205, www.euro-betriebsrat.de/pdf/kn.pdf Blanke, T. (2006): Europäisches Betriebsräte-Gesetz. Europäische Mitbestimmung, Baden-Baden

Carley, M./Hall, M. (2006): European Works Councils and transnational restructuring. Luxembourg: Office for Official Publications of the European Communities

Europäische Kommission (2008): Europäische Betriebsräte: Anhörung der europäischen Sozialpartner zur Änderung der Richtlinie 94/45/EG des Rates vom 22. September 1994 über die Einsetzung eines Europäischen Betriebsrats oder die Schaffung eines Verfahrens zur Unterrichtung und Anhörung der Arbeitnehmer in gemeinschaftsweit operierenden Unternehmen und Unternehmensgruppen, ec.europa.eu/employment_social/ labour_law/docs/2008/ewc_consultation2_de.pdf
Kerckhofs, P. (2006): European works councils: Facts and figures, Brussels Pas, I./Kerckhofs, P. (2006): European Works Councils Database, CDROM, Brussels

Stiftung Familienunternehmen (Hrsg.) (2006a): Länderindex der Stiftung Familienunternehmen Stuttgart, www.familienunternehmen.de/media/ public/pdf/studie_web2006_04_25.pdf

Stiftung Familienunternehmen (2006b): Standortnachteil für deutsche Familienunternehmen, Pressemitteilung vom 3.4., Berlin Wallau, F./Haunschild, L./Hauser, H.-E./Wolter, H.-J. (2007): Die volkswirtschaftliche Bedeutung der Familienunternehmen, IfM-Materialien 172, Bonn, www.ifm-bonn.org/ergebnis/ifm-materialie-172.pdf 